Reinhard F. Hüttl, Josef Zens, Knut Kaiser und Oliver Bens

\title{
Brücken bauen mit moderner Erdsystemforschung - Wasser im Fokus internationaler Ressourcengerechtigkeit und des Schutzes vor Georisiken
}

In: Nida-Rümelin, Julian / Daniels, Detlef von / Wloka, Nicole (Hrsg.): Internationale Gerechtigkeit und institutionelle Verantwortung. - ISBN: 978-3-11-061586-9. - Berlin: De Gruyter, 2019.

S. 357-368

(Forschungsberichte / Interdisziplinäre Arbeitsgruppen, Berlin-Brandenburgische Akademie der Wissenschaften ; 41) 
Reinhard F. Hüttl, Josef Zens, Knut Kaiser und Oliver Bens Brücken bauen mit moderner Erdsystemforschung - Wasser im Fokus internationaler Ressourcengerechtigkeit und des Schutzes vor Georisiken

\section{Prolog}

Wasser ist nicht nur der Grundstoff allen irdischen Lebens, sondern auch die Grundlage unserer modernen Maßeinheiten. Im Jahr 1775 wurde das Gramm definiert als die Masse eines Kubikzentimeters flüssigen Wassers bei einer Temperatur, wenn Eis schmilzt. ${ }^{1}$ Das spätere Ur-Kilogramm wurde erst anhand dieser Einheit festgelegt. So gilt bis heute, dass ein Liter Wasser ein Kilogramm wiegt und eine Tonne Wasser einem Kubikmeter entspricht. Und wer Wohlstand und Entwicklung auf der Erde messen will, zieht die Verfügbarkeit von sauberem Wasser als einen wichtigen Indikator heran. Das Thema internationale Ressourcengerechtigkeit umfasst daher auch als zentralen Bestandteil Wasser - dessen dargebotene Menge ebenso wie die Verfügbarkeit für die menschliche Nutzung, die Verfügungsgewalt darüber und die tatsächliche Verteilung. Auch der Schutz vor hydrologischen Extremereignissen - Dürre ebenso wie Flut - ist eine Ressourcen- und eine Gerechtigkeitsfrage. Hier ist nicht Wasser, sondern Wissen die entscheidende Ressource. Eine Kernaufgabe der internationalen und der nationalen Politik ist es, diese Themen zu verhandeln. Die Wissenschaft, insbesondere die Geoforschung, leistet hierbei einen fundamentalen Beitrag, indem sie Daten erhebt und anhand von diesen Anwendungsoptionen entwickelt.

Der folgende Beitrag zeigt anhand von vier Beispielen zum Thema Ressource Wasser, was die moderne Geoforschung zur Ressourcengerechtigkeit beitragen kann.

1 „Décret relatif aux poids et aux mesures du 18 germinal an 3 (7 avril 1795)“, zitiert nach Wikipedia: https://en.wikipedia.org/wiki/Litre\#cite_note-decree-5, abgerufen am 21.11.2018.

Ә OpenAccess. ๑ 2019 Reinhard F. Hüttl, Josef Zens, Knut Kaiser und Oliver Bens, publiziert von De Gruyter. (c) BY-NC-SA Dieses Werk ist lizenziert unter der Creative Commons Attribution-NonCommercial-ShareAlike 4.0 Lizenz. https://doi.org/10.1515/9783110615876-021 


\section{Grundwasser: Die unsichtbare Ressource wird aus dem All vermessen}

Flüsse boten immer schon begehrte Orte, um dort zu siedeln. Das lag nicht nur an den Transportmöglichkeiten, die ein großes Fließgewässer bietet, sondern auch am Wasser als Nahrungs- und Energieressource (Kaiser et al. 2012). Als in Zeiten der Industrialisierung und der zunehmenden Bevölkerungsdichte in den Städten die Oberflächengewässer immer stärker verunreinigt wurden, gewann Grundwasser - da in der Regel äußerst sauber - zunehmend an Bedeutung. Die Nutzung von wasserleitenden Schichten, sogenannten Aquiferen, spielt eine entscheidende Rolle in der Stadt- und Regionalentwicklung und auch bei der Besiedlung von Regionen, die ohne Brunnen unbewohnbar wären.

Eine Stadt wie Berlin fördert pro Tag rund eine halbe Million Kubikmeter frisches Wasser. ${ }^{2}$ Die Bundeshauptstadt nutzt dazu vielfach sogenanntes Uferfiltrat, also Wasser, das aus Seen und Flüssen in den Untergrund sickert und während der Passage durch die Bodenbakterien und Gesteinsschichten gereinigt wird (Schug et al. 2014). Insbesondere in sehr trockenen Regionen ist das anders: Da stammt der größte Teil der Reserven aus fossilen Grundwasserleitern. Die unterirdischen Schichten wurden vor vielen Jahrtausenden - teilweise noch während der letzten Eiszeit - gefüllt. Die derzeitige Nutzung ist dann keine nachhaltige, sondern gleicht eher dem Abbau von Salz, Erz oder Kohle. Das heißt aber auch, dass diese Aquifere sich langsam entleeren und auf absehbare Zeit nicht mehr wieder aufgefüllt werden (Gleeson et al. 2012).

Die Nutzung von fossilem Grundwasser im Sinne eines „Grundwasserabbaus“ ist ganz unmittelbar mit Ressourcengerechtigkeit verbunden, da Grundwasser führende Schichten sich nicht an Staats- oder Eigentumsgrenzen orientieren. Eine Überwachung der Grundwasservorräte ist jedoch schwer. Bohrungen sind teuer und liefern nur punktuelle Werte. Die Satellitenmission GRACE (Gravity Recovery and Climate Experiment) und ihre Nachfolgemission GRACE-FO (FO steht für Follow On) des Deutschen GeoForschungsZentrums GFZ und der US-Weltraumbehörde NASA schufen hier Abhilfe. ${ }^{3}$

2 Angabe der Berliner Wasserbetriebe, http://www.bwb.de/de/941.php, abgerufen am 21.11. 2018.

3 Das Prinzip von GRACE und GRACE-FO: Zwei Satelliten umkreisen die Erde in rund 500 Kilometern Höhe und fliegen in einem Abstand von etwa 220 Kilometern. Befindet sich unter der Flugbahn eine große Masse, zum Beispiel der Mount Everest, dann zieht diese Masse den ersten der herannahenden Satelliten mehr an als den hinterherfliegenden. Der Abstand vergrößert sich. Ist der erste über die Masse hinweggeflogen, zieht sie ihn wiederum an, diesmal aber nach 
Die NASA schreibt dazu:

Durch die Beobachtung von Änderungen im Erdschwerefeld können Wissenschaftler Veränderungen der Wassermenge in einer Region abschätzen, die Veränderungen in der Schwerkraft verursachen. GRACE liefert einen mehr als 10 Jahre langen Datensatz für wissenschaftliche Analysen. Das macht einen großen Unterschied für Wissenschaftler und Wassermanager, die Trends in Bezug auf den Verbrauch unserer Ressourcen auf lange Sicht verstehen wollen. GRACE hat Daten über einige der größten Aquifere der Welt erhoben und darüber, wie sich ihr Wasserspeicher verändert (Rodell/Famiglietti 2001; Yeh et al. 2006; Rodell et al. 2007). Mit Hilfe von Schätzungen der Veränderungen bei der Schnee- und Oberflächenfeuchte können Wissenschaftler eine Veränderung des Grundwasservolumens über einen bestimmten Zeitraum genau berechnen. Eine Studie von Rodell et al. (2009) im Nordwesten Indiens nutzte Veränderungen des terrestrischen Wasserspeichers von GRACE und simulierte Bodenwasservariationen aus einem datenintegrierenden hydrologischen Modellsystem, um zu zeigen, dass Grundwasser in den indischen Bundesstaaten Rajasthan, Punjab und Haryana (einschließlich Delhi) mit einer mittleren Rate von 4,0 (+/ - 1,0) cm pro Jahr abgebaut wird. Das entspricht 17,7 (+/- 4,5) Kubikkilometern pro Jahr. Während des Untersuchungszeitraums von August 2002 bis Oktober 2008 entsprach der Grundwasserabbau einem Nettoverlust von 109 Kubikkilometern Wasser, was der doppelten Kapazität von Indiens größtem Oberflächenwasserreservoir entspricht. ${ }^{4}$

Zum Vergleich: Berlin verbraucht pro Jahr rund 0,2 Kubikkilometer Wasser ${ }^{5}$ und könnte mit der oben für Indien angegebenen Menge also - gleichbleibenden Verbrauch vorausgesetzt - mehr als 500 Jahre lang versorgt werden.

Ähnliche Arbeiten gibt es für das Central Valley in Kalifornien, wo das Grundwasser häufig den größten Teil des für die Bewässerung benötigten Wassers liefert (Famiglietti et al. 2011). Das Tal wird intensiv landwirtschaftlich genutzt. GRACE-Daten zeigten, dass die kalifornischen Becken des Sacramento und des San Joaquin River zwischen 2003 und 2010 insgesamt 30,9 Kubikkilometer Grundwasser verloren - das entspricht 150 Jahren Berliner Wasserversorgung. Hydrologische Modellrechnungen legen nahe, dass der Großteil dieser Verluste auf den Grundwasserabbau im Central Valley zurückzuführen war (Abb. 1).

Grundwasserspeicher in Flusseinzugsgebieten können allerdings auch zu voll werden. Ist das der Fall, steigt das Risiko eines Hochwassers, da die unterirdischen Speicher den Regen nicht mehr aufnehmen können. Und auch hier gilt: Flüsse und die dazugehörigen Täler und Grundwasserspeicher halten sich nicht an Landesgrenzen. Hochwasserschutzmaßnahmen ebenso wie Frühwarnsysteme

„hinten“: Sie bremst ihn also minimal ab. Zur selben Zeit wird der zweite Satellit von der Masse angezogen, also beschleunigt. Die Folge: Der Abstand verringert sich. Aus diesen Abstandsänderungen der Satelliten lassen sich Rückschlüsse auf die jeweils überflogene Masse ziehen.

4 https://grace.jpl.nasa.gov/applications/groundwater/ (Stand 19.09.2018; eigene Übersetzung).

5 Eigene Berechnung, basierend auf Angaben der Berliner Wasserbetriebe (vgl. Fußnote 2). 
A

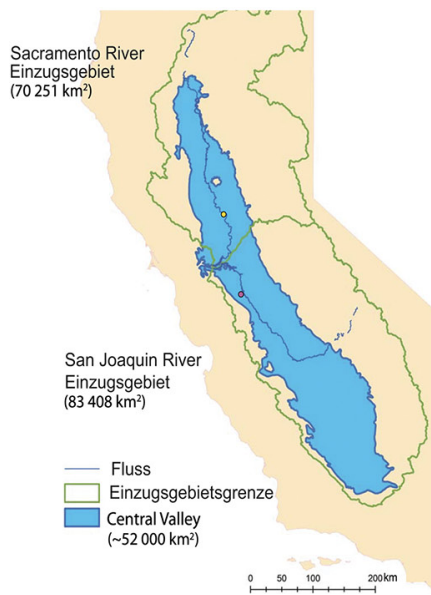

B

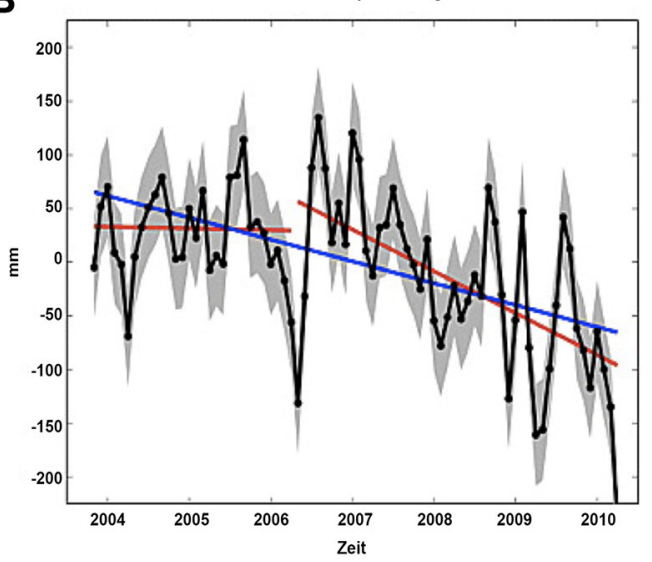

Abb. 1: Die Flusseinzugsgebiete des Sacramento und des San Joaquin River im Central Valley in Kalifornien (A) mit monatlichen Grundwasservorratsschwankungen für diese Flusseinzugsgebiete (in mm) im Zeitraum Oktober 2003 bis März 2010, ermittelt durch GRACE-Daten (B; auf Deutsch umbeschriftet nach Famiglietti et al. 2011). Die Fehlerbandbreite der Monatsdaten ist als graues Band dargestellt. Die blaue Linie repräsentiert den Gesamttrend über eine Beobachtungsdauer von 78 Monaten; die rote Linie repräsentiert kürzere Trends in den Perioden Oktober 2003 bis März 2006 und April 2006 bis März 2010. Erkennbar wird seit 2006/2007 eine erhebliche Abnahme der Grundwasservorräte.

müssen sich an den naturräumlichen Gegebenheiten orientieren und nicht an politischen Grenzen.

\section{Risikoanalysen für ein effizientes Hochwasserrisikomanagement}

Hochwasser führen oft zu sehr hohen Schäden. ${ }^{6}$ So gab es im Juni 2013 in Mitteleuropa ein Hochwasser-Extremereignis (u. a. an Elbe und Donau), das allein in Deutschland 14 Todesopfer und Schäden in Höhe von 8 Milliarden Euro verursachte (DKKV 2015). Für ein effizientes Hochwasserrisikomanagement sind daher Risikoanalysen unerlässlich. Dazu gehört die Abschätzung der Hochwassergefährdung und der möglichen Schäden. Allerdings gibt es bei der Schadensmodellierung noch hohe Unsicherheiten. Um diese zu minimieren, sind empirische

6 Die folgenden Absätze zur Hochwasserschadensmodellierung sind Kreibich et al. (2018) entnommen. 
Daten ein Schlüsselelement. Sie helfen beim Erkennen der komplexen Zusammenhänge unterschiedlichster Einflussfaktoren. Kreibich et al. (2018) haben seit dem Elbe-Hochwasserereignis im August 2002 für alle großen, schadensträchtigen Hochwasserereignisse in Deutschland Daten über die entstandenen Schäden und möglichst viele potenziell wichtige Einflussfaktoren durch Befragungen von betroffenen Privathaushalten und Unternehmen erhoben. Auf diese Weise ist eine weltweit einzigartige Datenbank mit objektspezifischen Hochwasserschäden entstanden. Sie ist singulär im Hinblick auf die Detailliertheit der enthaltenen Informationen und die Anzahl der enthaltenen Schadensfälle. Dazu kommt das Verfahren des maschinellen Lernens zur Anwendung, um die Schädigungsprozesse besser $\mathrm{zu}$ verstehen und die wichtigsten schadensbeeinflussenden Variablen $\mathrm{zu}$ identifizieren.

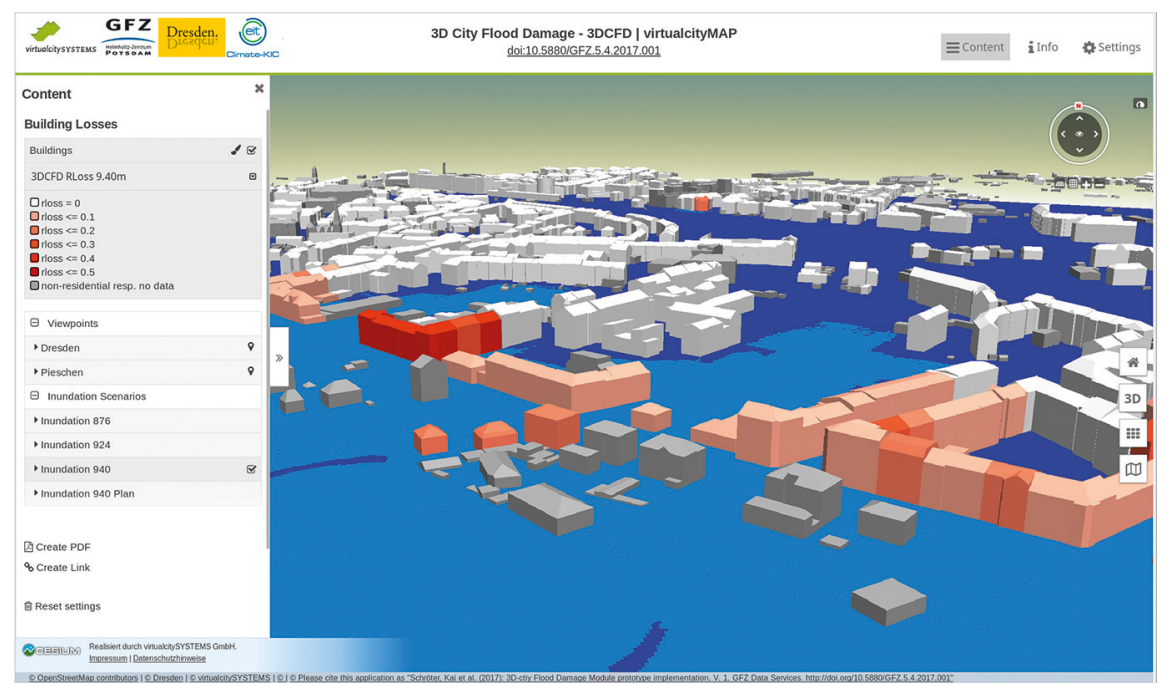

Abb. 2: Graphische Bildschirmoberfläche eines am Deutschen GeoForschungsZentrum GFZ gemeinsam mit Partnern entwickelten Hochwasserschaden-Modellierungsprogramms auf Basis von 3D-Stadtmodellen (nach Schröter et al. 2018)

Beispielsweise wurden auf Basis von insgesamt 783 Schadensdaten von urbanen Sturzflutereignissen zunächst 55 Variablen abgeleitet, die einen möglichen Einfluss auf den Wohngebäudeschaden haben. Mittels vier verschiedener maschineller Lernverfahren wurden einerseits die wichtigsten Variablen ermittelt, die bestimmen, ob ein Schaden aufgetreten ist, und andererseits diejenigen Variablen, die die Höhe des Schadens bestimmen. Es stellte sich heraus, dass neben dem Wasserstand vor allem Widerstandsfaktoren wie die Haushaltsgröße oder das Wissen über 
Hochwassergefährdung darüber entscheiden, ob ein Gebäudeschaden entsteht. Die Höhe des Schadens wird dagegen in erster Linie durch Einwirkungsfaktoren wie Wasserstand, Dauer der Überflutung und Kontamination beeinflusst. Zudem wird das maschinelle Lernen eingesetzt, um Schädigungsprozesse $\mathrm{zu}$ modellieren (Abb. 2). Informationen darüber sind für die Risikokommunikation, aber auch für die Entscheidungsfindung im Risikomanagement, d. h. bei der Hochwasserschutzund Hochwasservorsorgeplanung von enormer Bedeutung.

\section{Wasser in Zentralasien: Ungleich verteilte Ressourcen werden quantifiziert}

Daten sind auch von entscheidender Bedeutung beim Wassermanagement in den fünf zentralasiatischen Staaten Kirgisistan, Usbekistan, Tadschikistan, Turkmenistan und Kasachstan. Die ehemaligen Sowjetrepubliken sind mit wasserbezogenen Problemen konfrontiert, die ein hohes Konfliktpotenzial aufweisen (Hüttl et al. 2016). Zudem ist Wasser dort der Schlüsselfaktor für die wirtschaftliche Entwicklung. Die Ressource ist jedoch ungleich verteilt. Nur verlässliche Daten, die für alle zentralasiatischen Staaten zugänglich sind, können Vertrauen aufbauen und zu nachhaltigen Wassermanagementstrategien führen, die von allen Staaten akzeptiert werden.

Es gibt zwei große Flüsse in der Region: Amu Darja und Syr Darja. Die Staaten in den gebirgigen Oberläufen verfügen über reiche Wasservorkommen und nutzen diese hauptsächlich zur Energiegewinnung im Winter. Im Unterlauf dagegen wird das Wasser vor allem im Sommer zur Bewässerung von Ackerflächen benötigt. Mehrere Faktoren verschärfen die Konfliktsituation: Bewässerungsinfrastrukturen sind veraltet, Kanäle undicht und die landwirtschaftlichen Methoden allgemein ineffizient. Dazu kommt der Ausfall vieler hydrologischer Messstationen nach dem Zerfall der Sowjetunion. In der Folge versalzen die Böden und das Grundwasser in der Region. Die Entnahme großer Wassermengen aus Amu Darja und Syr Darja hat die Wüstenbildung verstärkt und letztlich zur Katastrophe des Austrocknens des Aralsees geführt (Breckle et al. 2011).

$\mathrm{Zu}$ den politischen und technischen Problemen kommt der Klimawandel hinzu. Er stellt einen erheblichen Unsicherheitsfaktor hinsichtlich der zukünftigen Dynamik von Wasserressourcen und wasserbedingten Katastrophen dar. Während der Rückzug der Gletscher im Tien Shan und Pamir seit den 1960er Jahren u. a. mit Hilfe von Satelliten-Daten rekonstruiert wurde (Fariotti et al. 2015; Abb. 3), müssen die Auswirkungen dieses Prozesses auf den Abfluss noch detailliert geklärt werden. Es wird erwartet, dass sich Häufigkeit, Saisonalität und 
Größenordnung von Wasserkatastrophen durch veränderte klimatische Faktoren ebenfalls verändern werden (Kenjabaev/Frede 2016). Hier kann die wissenschaftliche Forschung als Katalysator für die zwischenstaatliche Zusammenarbeit auf politischer und administrativer Ebene dienen. Darüber hinaus wird nur die Wissenschaft in der Lage sein, eine verlässliche Datenbasis zu liefern, wie sie für nachhaltige Entscheidungen in der Wasserwirtschaft benötigt wird.

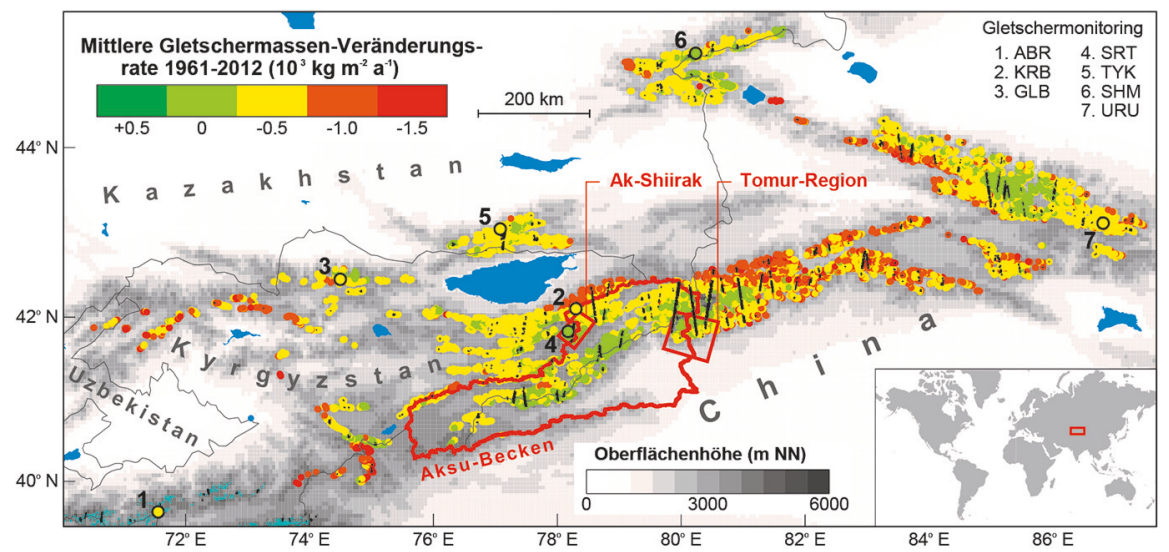

Abb. 3: Massenveränderungen von Gletschern im Tien Shan im Zeitraum 1961-2012 (mit Erlaubnis von Springer Nature, verändert nach Fariotti et al. 2015). Jeder Farbpunkt repräsentiert einen Gletscher. Die vorherrschenden gelben, orangen und roten Farben indizieren einen weitgehenden Schwund von Gletschern in dieser Zeit.

Aus diesem Grund hat das Auswärtige Amt gemeinsam mit zahlreichen nationalen und internationalen Partnereinrichtungen im Jahr 2008 das Projekt Central Asian Water (CAWa) gegründet. Dieses Vorhaben wurde im Herbst 2018 mit einer Konferenz in Almaty/Kasachstan abgeschlossen (Central Asia Water Project 2018). Es hat dazu beigetragen, eine solide wissenschaftliche Grundlage für das transnationale Wasserressourcenmanagement in Zentralasien $\mathrm{zu}$ schaffen. Innerhalb des CAWa-Netzwerks wurden in Zentralasien moderne hydrometeorologische Stationen mit hochentwickelten Sensoren installiert. Die Stationen senden ihre Daten über Echtzeit-Satellitenkommunikation an das Betriebszentrum des Überwachungsnetzes und an die teilnehmenden nationalen hydrometeorologischen Dienste. Die hydrometeorologischen Daten der neuen Stationen, die Daten der Fernerkundung und des globalen Navigationssatellitensystems GNSS sowie die Ergebnisse der wissenschaftlichen Studien werden allen Partnern über das Internet zugänglich gemacht. So wird CAWa zu einer verlässlichen Datenbasis über die Wasserressourcen in Zentralasien beitragen. Darüber hinaus werden die in 
CAWa gewonnenen Methoden und Erfahrungen in Schulungen an zentralasiatische Fachleute aus Wissenschaft und Verwaltung weitergegeben und für die Entwicklung von Lehrplänen an Universitäten und anderen Hochschulen genutzt. Damit wird ein wichtiger Beitrag zur Entwicklung einer möglichst nachhaltigen „Wasser-Gerechtigkeit“ in dieser gesellschaftspolitisch schwierigen Region der ehemaligen zentralistisch organisierten Sowjetunion geleistet.

\section{Globaler Wandel und seine Auswirkungen auf Regionen}

Der Globale Wandel wirkt sich jedoch nicht nur auf die besonders empfindlichen Regionen in den zentralasiatischen Hochgebirgen und ihren Vorländern aus. Vielmehr betrifft er viele Weltregionen, aber auch unsere unmittelbare Umgebung in Deutschland. Entsprechend der Mehrdimensionalität globaler Veränderungsprozesse in Umwelt, Wirtschaft und Demographie wurden und werden hier verschiedene Projekte realisiert, die diesen thematischen Facetten mit einem Schwerpunkt oder Teilschwerpunkt Wasser Rechnung tragen und auf besonders sensible bzw. vulnerable Regionen fokussieren (z. B. Hüttl et al. 2008; Hüttl et al. 2011; Grünewald et al. 2012; Hüttl/Bens 2012).

So wurden im Rahmen der Initiative TERrestrial ENvironmental Observatories - oder kurz TERENO - seit 2008 vier regionale Observatorien eingerichtet, um in Deutschland Umweltveränderungen inklusive des Klimawandels zu erfassen. Die TERENO-Observatorien befinden sich in der Eifel und der Niederrheinischen Bucht, im Harz und im mitteldeutschen Tiefland, in den bayerischen Alpen und im Alpenvorland sowie im nordostdeutschen Tiefland. Alle vier Gebiete sind mit einer Kombination aus kontinuierlich aufzeichnenden Messeinrichtungen sowie boden- und luftgestützten Fernerkundungstechnologien ausgerüstet. Mess- und Beprobungskampagnen ergänzen dieses System. Diese zunächst bis zum Jahre 2025 angelegte Infrastrukturmaßnahme verfolgt das Ziel, Veränderungen in der Geo-, Hydro-, Bio- und unteren Atmosphäre sowie der Anthroposphäre auf unterschiedlichen räumlichen und zeitlichen Skalen zu erfassen. Dieser Ansatz ermöglicht es, Wirkungszusammenhänge besser zu verstehen und effektive Präventions-, Vermeidungs- und Anpassungsstrategien insbesondere mit Fokus Klimawandel zu entwickeln.

Das nordostdeutsche Tiefland zählt zu den potenziell am stärksten vom Klimawandel betroffenen Regionen in Mitteleuropa, wo relative Trockenheit und die Zunahme von hydrometeorologischen Extremereignissen zu den größten Herausforderungen zählen (Kaiser et al. 2010; Germer et al. 2011; Hüttl/Bens 2012; 
Abb. 4). Das Observatorium Nordostdeutsches Tiefland - „TERENO-Nordost“ untersucht deshalb schwerpunktmäßig den Einfluss des Klima- und Landnutzungswandels auf terrestrische und aquatische Ökosysteme (Heinrich et al. 2018). Dieses Observatorium umfasst derzeit fünf Standorte, darunter ein Teileinzugsgebiet der Ucker, den Müritz-Nationalpark, den Tiefen See auf der Mecklenburgischen Seenplatte, den Kalibrations- und Validationsstandort Demmin sowie ein Moorgebiet an der Ostseeküste bei Rostock.

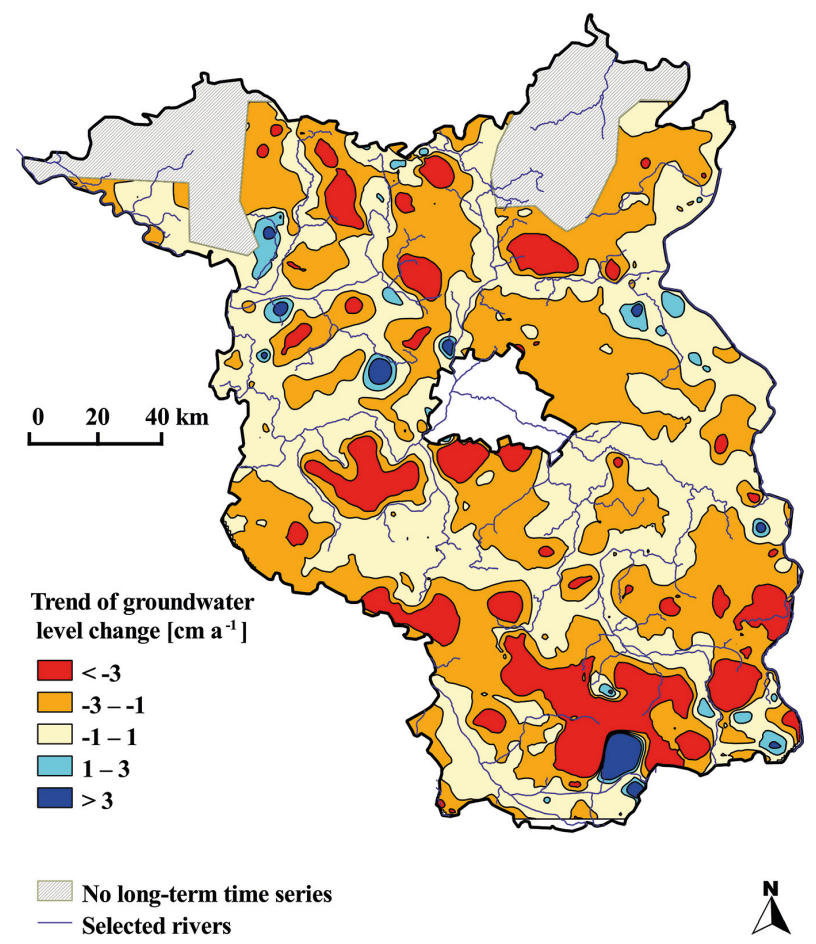

Abb. 4: Trend des Wasserspiegels des obersten unbedeckten Grundwasserkörpers im Bundesland Brandenburg von 1976 bis 2005, interpoliert anhand der Daten von 1183 Grundwasserpegeln (aus Germer et al. 2011). Weite Teile Brandenburgs erfuhren demnach in diesem Zeitraum eine erhebliche Absenkung des Grundwasserspiegels.

Die komplexen Zusammenhänge und Rückkopplungsmechanismen der verschiedenen Teile der terrestrischen und aquatischen Systeme erfordern einen interdisziplinären Forschungsansatz. Wichtige Systemvariablen, die im Rahmen von TERENO-Nordost untersucht werden, sind deshalb Verlagerungs- und Austauschprozesse von Wasser, Materie und Energie im Grundwasser-Boden-Vegetation-Atmosphäre-System und langfristige Veränderungen des Aufbaus und der 
Funktion von Mikroorganismen-Gesellschaften sowie der Flora und Fauna, die mit ausreichender zeitlicher und räumlicher Auflösung bestimmt werden müssen. Damit werden naturwissenschaftliche Fragestellungen bearbeitet wie z. B.: Welchen Einfluss haben die aktuellen und künftigen Klimaänderungen auf Grundwasser, Oberflächengewässer, Böden und Vegetation? Oder: Welche direkten Einflüsse haben Veränderungen der Boden- und Landnutzung auf den Wasserhaushalt, die Bodenfruchtbarkeit, die Biodiversität und das regionale Klima? Fragen, die auch gesellschaftspolitisch hochrelevant sind.

\section{Epilog}

Die hier für die Ressource Wasser kurz skizzierten Fallbeispiele dokumentieren, dass Ressourcengerechtigkeit insbesondere unter den Bedingungen des Globalen Wandels eine enorme Herausforderung darstellt, die die Politik zu verhandeln hat: Gibt es Vorrang bei der Nutzung von Wasser, etwa zu Kühlzwecken für Kraftwerke oder zur Bewässerung in der Landwirtschaft? Gibt es Schutzinteressen bei Ökosystemen, die Nutzungsinteressen - Wasserwirtschaft, Land- und Forstwirtschaft, Energiewirtschaft, Siedlungs- und Infrastrukturentwicklung - entgegenstehen? Wie lassen sich also allgemein die sensiblen Wasserressourcen in einer Region mit den Erfordernissen der ökonomischen Entwicklung unter Wahrung der ökologischen Belange in Einklang bringen? Im Zuge einer vorausschauenden Politik kommt dem Vorsorgeprinzip und dem Ausgleich von Interessen eine besondere Rolle im Rahmen einer Ressourcengerechtigkeit zu. Aus der Geoforschung werden zu vielen relevanten Fragen Grundlagen für Handlungsoptionen sowie für evidenzbasierte Entscheidungen bereitgestellt. In diesem Zusammenhang haben auch die Interdisziplinären Arbeitsgruppen der BerlinBrandenburgischen Akademie der Wissenschaften „LandInnovation“ (Hüttl et al. 2008), „Globaler Wandel - Regionale Entwicklung“ (Hüttl et al. 2011) sowie „Gesellschaft - Wasser - Technik“ (Hüttl et al. 2016) anhand von Beispielregionen in Nordostdeutschland, dem Nahen Osten und Zentralasien wichtige Beiträge geleistet.

\section{Bibliographie}

Breckle, Siegmar W./Wucherer, Walter/Dimeyeva, Liliya A./Ogar, Nathalia P. (Hrsg.) (2011): Aralkum - A Man-Made Desert. The Desiccated Floor of the Aral Sea (Central Asia) (= Ecological Studies 218). Berlin, New York: Springer. 
Central Asia Water Project (2018): „Proceedings Paper. International Symposium on Water and Land Resources in Central Asia, 9-11 October 2018, Almaty, Kazakhstan“. Potsdam: Deutsches GeoForschungsZentrum GFZ.

DKKV - Deutsches Komitee Katastrophenvorsorge e.V. (Hrsg.) (2015): Das Hochwasser im Juni 2013: Bewährungsprobe für das Hochwasserrisikomanagement in Deutschland (= DKKV-Schriftenreihe 53). Bonn: DKKV.

Famiglietti, James S./Lo, Minhui/Ho, Sing L./Bethune, James/Anderson, Ray G./Syed, Tajdarul H./Swenson, Sean C./de Linage, Caroline R./Rodell, Matthew (2011). „Satellites Measure Recent Rates of Groundwater Depletion in California’s Central Valley“. In: Geophysical Research Letters 38, L03403.

Farinotti, Daniel/Longuevergne, Laurent/Moholdt, Geir/Düthmann, Doris/Mölg, Thomas/Bolch, Tobias/Vorogushyn, Sergiy/Güntner, Andreas (2015): „Substantial Glacier Mass Loss in the Tien Shan over the Past 50 Years“. In: Nature Geoscience 8, S. 716-722.

Germer, Sonja/Bens, Oliver/Hüttl, Reinhard (Hrsg.) (2011): „Global Change: Challenges for Regional Water Resources“. In: Die Erde 142, Nr. 1-2, S. 1-208.

Grünewald, Uwe/Bens, Oliver/Hüttl, Reinhard/Kaiser, Knut/Knierim, Andrea (Hrsg.) (2012): Wasserbezogene Anpassungsmaßnahmen an den Landschafts- und Klimawandel. Stuttgart: Schweizerbart.

Gleeson, Tom/Wada, Yoshihide/Bierkens, Marc F.P./van Beek, Ludovicus P.H. (2012): „Water Balance of Global Aquifers Revealed by Groundwater Footprint“. In: Nature 488, S. $197-200$.

Heinrich, Ingo/Balanzategui, Daniel/Bens, Oliver/Blasch, Gerald/Blume, Theresa/Böttcher, Falk/Borg, Erik/Brademann, Brian/Brauer, Achim/Conrad, Christopher/Dietze, Elisabeth/Dräger, Nadine/Fiener, Peter/Gerke, Horst H./Güntner, Andreas/Heine, Iris/Helle, Gerd/Herbrich, Marcus/Harfenmeister, Katharina/Heußner, Karl-Uwe/Hohmann, Christian/Itzerott, Sibylle/Jurasinski, Gerald/Kaiser, Knut/Kappler, Christoph/Koebsch, Franziska/Liebner, Susanne/Lischeid, Gunnar/Merz, Bruno/Missling, Klaus D./Morgner, Markus/Pinkerneil, Sylvia/Plessen, Birgit/Raab, Thomas/Ruhtz, Thomas/Sachs, Torsten/Sommer, Michael/Spengler, Daniel/Stender, Vivien/Stüve, Peter/Wilken, Florian (2018): „Interdisciplinary Geo-ecological Research across Time Scales in the Northeast German Lowland Observatory (TERENO-NE)“. In: Vadose Zone Journal 17, S. 1- 25.

Hüttl, Reinhard/Bens, Oliver/Plieninger, Tobias (Hrsg.) (2008): Zur Zukunft ländlicher Räume: Entwicklungen und Innovationen in peripheren Regionen Nordostdeutschlands (= Forschungsberichte der Interdisziplinären Arbeitsgruppen der Berlin-Brandenburgischen Akademie der Wissenschaften 20). Berlin: Akademie Verlag. Hüttl, Reinhard/Emmermann, Rolf/Germer, Sonja/Naumann, Matthias/Bens, Oliver (Hrsg.) (2011): Globaler Wandel und regionale Entwicklung - Anpassungsstrategien in der Region Berlin-Brandenburg. Berlin, New York: Springer.

Hüttl, Reinhard/Bens, Oliver (Hrsg.) (2012): Georessource Wasser - Herausforderung Globaler Wandel: Beiträge zu einer nachhaltigen Wasserressourcenbewirtschaftung in Deutschland. Berlin, New York: Springer.

Hüttl, Reinhard/Bens, Oliver/Bismuth, Christine/Hoechstetter, Sebastian (Hrsg.) (2016): Society - Water - Technology: A Critical Appraisal of Major Water Engineering Projects. Berlin, New York: Springer. 
Kaiser, Knut/Libra, Judy/Merz, Bruno/Bens, Oliver/Hüttl, Reinhard (Hrsg.) (2010): Aktuelle Probleme im Wasserhaushalt von Nordostdeutschland: Trends, Ursachen, Lösungen (= Scientific Technical Report 10/10). Potsdam: Deutsches GeoForschungsZentrum.

Kaiser, Knut/Merz, Bruno/Bens, Oliver/Hüttl, Reinhard (Hrsg.) (2012): Historische Perspektiven auf Wasserhaushalt und Wassernutzung in Mitteleuropa (= Cottbuser Studien zur Geschichte von Technik, Arbeit und Umwelt 38). Münster: Waxmann.

Kenjabaev, Shavkat/Frede, Hans-Georg (2016): „Irrigation Infrastructure in Fergana Today: Ecological Implications - Economic Necessities“. In: Reinhard Hüttl/Oliver Bens/Christine Bismuth/Sebastian Hoechstetter (Hrsg.): Society - Water - Technology: A Critical Review of Major Water Engineering Projects. Berlin, New York: Springer, S. 129-148.

Kreibich, Heidi/Schröter, Kai/Shprits, Yuri/Bedford, Jonathan/Tilmann, Frederik (2018): „Maschinelles Lernen verbessert Gefährdungs- und Risikoanalysen von Naturgefahren“. In: System Erde 8, S. 10-17.

Rodell, Matthew/Famiglietti, James S. (2001): „An Analysis of Terrestrial Water Storage Variations in Illinois with Implications for the Gravity Recovery and Climate Experiment (GRACE)“. In: Water Resources Research 37, S. 1327-1340.

Rodell, Matthew/Chen, Jianli/Kato, Hiroko/Famiglietti, James S./Nigro, Joe/Wilson, Clark R. (2007): „Estimating Groundwater Storage Changes in the Mississippi River Basin (USA) Using GRACE“. In: Hydrogeology Journal 15, S. 159-166.

Rodell, Matthew/Velicogna, Isabella/Famiglietti, James S. (2009): „Satellite-based Estimates of Groundwater Depletion in India“. In: Nature 460, S. 999-1002.

Schröter, Kai/Lüdtke, Stefan/Redweik, Richard/Meier, Jessica/Bochow, Mathias/Ross, Lutz/Nagel, Claus/Kreibich, Heidi (2018): „Flood Loss Estimation Using 3D City Models and Remote Sensing Data“. In: Environmental Modelling and Software 105, S. 118-131.

Schug Alexander/Kessinger, Bernd/Roeckner, Katja/Petrasch, Frank (2014): Berliner Wasser: Die Geschichte einer Lebensnotwendigkeit. Berlin: Vergangenheitsverlag.

Yeh, Pat J.-F./Swenson, Sean C./Famiglietti, James S./Rodell, Matthew (2006): „Remote Sensing of Groundwater Storage Changes in Illinois Using the Gravity Recovery and Climate Experiment (GRACE)“. In: Water Resources Research 42: W12203. 